\title{
Corneal topography by keratometry
}

\author{
W A Douthwaite, W T Evardson
}

\begin{abstract}
Aims-To investigate the ability of a telecentric keratometer to describe the asphericity and curvature of convex ellipsoidal surfaces and human corneas.

Methods-22 conicoidal convex surfaces and 30 human corneas were examined by conventional keratometry. Additional keratometric measurements were made when the surface was tilted in the horizontal plane relative to the instrument optical axis. This resulted in a series of radius measurements derived from different regions of the surface. These measurements were used to determine the apical radius and the $p$ value of the horizontal meridian of each surface. The results were compared with those derived from measurements using the EyeSys videokeratoscope and form Talysurf analysis. The method was repeated on 30 human corneas and the results compared with those of a videokeratoscope.

Results-For the aspheric buttons, the keratometric and the EyeSys results tended to give higher values for both apical radius and the $p$ values than those of the Talysurf analysis. The best agreement was between the Talysurf and the keratometer where the results were not significantly different. For the human corneas, the apical radii were significantly different comparing the keratometer with the videokeratoscope but the $p$ values were not significantly different.

Conclusion-The keratometric method for assessing curvature and asphericity appears to hold promise as a method for quantifying the corneal topography. (Br f Ophthalmol 2000;84:842-847)
\end{abstract}

The measurement of corneal topography has, in recent years, become a topic of interest to both ophthalmologists and optometrists. Detailed knowledge of corneal topography provides a tool for early detection and diagnosis of corneal pathology as well as a method for defining the optical characteristics of the anterior corneal surface. ${ }^{12}$ The recent ban on the use of contact lens trial sets has posed problems for the contact lens practitioner. In the past, the optimum fit of a lens could be conveniently assessed by observing the behaviour of trial set lenses on the eye of the patient. The keratometer gives some guidance for deriving an appropriate contact lens specification but the fact that the keratometer provides only a single radius of curvature for each of the two principal meridians of the cornea means that this guidance lacks precision. Quantifica- tion of the corneal topography allows for a subsequent calculation of the optimum back surface specification of a contact lens without the use of trial set lenses. ${ }^{3}$ The precision is likely to be as good as that achieved when trial lenses are used, with a significant saving of patient chair time.

Numerous methods have been proposed for measuring corneal topography starting with the placido disc in 1880 . The placido disc target, which consists of a number of concentric rings, is utilised in the photokeratoscope and the videokeratoscope (VK).

Bonnet $^{5}$ used raster stereography where a grid pattern was projected onto the cornea and the resulting distorted image of the grid could be used to investigate the corneal topography. A more detailed description is given by Arffa et al. ${ }^{6}$ A commercially available instrument called the PAR technology corneal topography system has been investigated by Belin et $a l,{ }^{78}$ who found the instrument to be both accurate and repeatable. A more recent clinical instrument devised to measure corneal topography is the Orbscan (Orbtech of Salt Lake City, UT, USA). This uses a scanning slit beam to measure the topography of both the anterior and posterior corneal surfaces along with the corneal thickness profile.

The problem with all these instruments is that their sophistication inevitably induces a penalty in terms of cost. An assessment of corneal topography using a simple instrument like the keratometer provides the possibility of topographical assessment for the clinician with access to only basic instrumentation.

Bonnet and Cochet $^{9}$ developed a topographical keratometer that used small mires to make peripheral keratometric measurements. If there is only a small change in the mire image size with change in corneal radius of curvature then the instrument sensitivity will be poor. This is a problem for small mire keratometry. It is also a problem for the smaller rings of the VK.

Wilms and Rabbetts ${ }^{10}$ suggested making peripheral corneal measurements by displacing the fixation direction along the plane of measurement. They called this technique topometry. Mathematical manipulation of the results produced an estimate of the surface asphericity but this involved a number of assumptions and approximations that make the final result questionable.

We decided to investigate the Wilms and Rabbets method. As an alternative to using their mathematical conversion to determine the surface asphericity, we decided to use calibrated aspherical surfaces. The calculations can then incorporate correction factors to convert an approximate into an accurate result 
based on comparisons with the actual specification of the aspherical surfaces.

\section{Method}

Twenty two conicoidal convex/plano buttons were sent to the Rank Taylor Hobson Calibration and Measurement Laboratory for form Talysurf analysis. This laboratory is part of the National Measurement Accreditation Service that provides facilities for checking or calibrating instruments and other test equipment against recognised international standards. The resolution is quoted as $20 \mathrm{~nm}$ or approximately $1 / 32$ of the wavelength of helium neon laser light. Relative to the best fit arc, the accuracy is claimed to be within two parallel planes, having a separation of $0.1 \mu \mathrm{m}$ over a $20 \mathrm{~mm}$ traverse after the removal of the best fit reference line. Two measurements were made on each surface-one parallel to an engraved line on the underside of the aspheric button with the other in the orthogonal meridian. Both measurements were made over the central $10 \mathrm{~mm}$ of the surface. This analysis allowed for the calculation of the surface apical radius $\left(r_{\mathrm{o}}\right)$ and the $\mathrm{p}$ value. The apical radius indicates the curvature and the $\mathrm{p}$ value indicates the asphericity. A spherical surface has a $p$ value of unity and a parabolic surface has a $\mathrm{p}$ value of zero.

The results of the Talysurf analysis are assumed to give an accurate assessment of the conicoidal surfaces and this provides a datum against which the results of any other method can be compared. The apical radii of the surfaces ranged from 7.131 to $8.203 \mathrm{~mm}$ and the $\mathrm{p}$ values ranged from -0.019 to 0.993 along the engraved meridian. The results for the orthogonal meridian were very similar.

The Zeiss Jena telecentric keratometer (model 110) was used to measure the surface curvature of the engraved meridian of each conicoidal button by conventional keratometry with the engraved meridian oriented along the horizontal. The surface was then tilted about a vertical axis and the keratometry was repeated. The geometry of the keratometer was used to

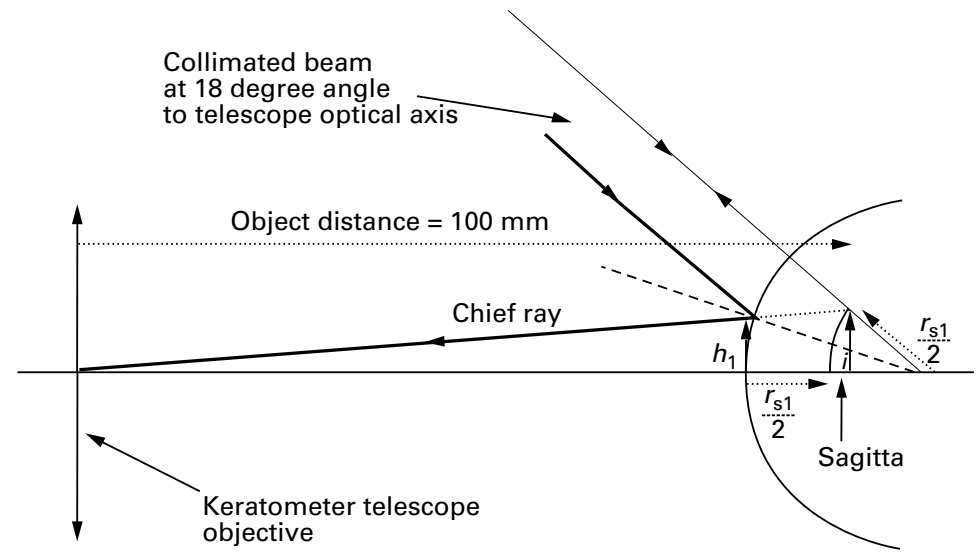

Figure 1 A keratometer with collimated mires is being used to measure the radius of curvature of a convex surface. For diagrammatic simplicity, the surface being examined is spherical. The chief ray, centred on the telescope objective lens, defines the region of the cornea which is being used to generate the mire image (size i) which is the object for the keratometer telescope. The corneal region being measured is centred on the point distance $h$ from the keratometer optical axis.

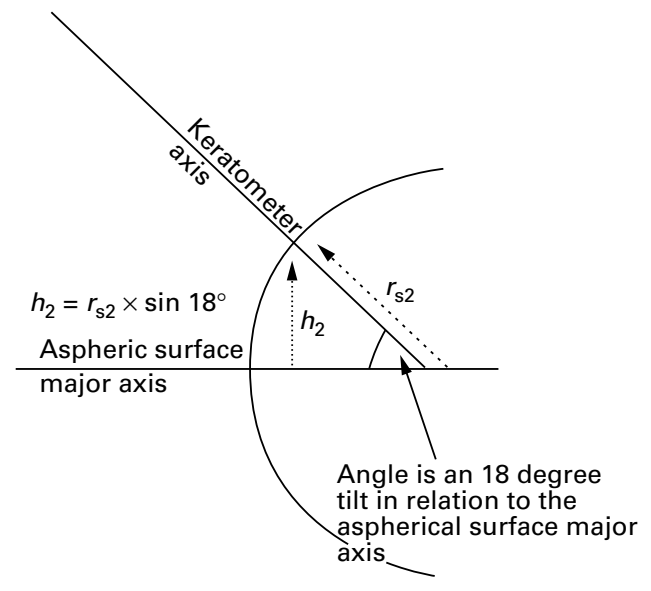

Figure 2 The keratometer is tilted $18^{\circ}$ in relation to the aspherical surface major axis. It is assumed that the instrument will measure the sagittal radius along the keratometer telescope optical axis. This is an oversimplification. A region of the surface further from the major axis and another region closer to the axis will form the mire images.

calculate the region of the cornea that was being measured in both cases as follows.

Figure 1 illustrates the situation for conventional keratometry measuring a spherical surface. The perpendicular distance $h_{1}$ defines the point on the cornea being measured. There is a corresponding point in the inferior cornea that gives an identical radius when measuring a rotationally symmetrical surface. The keratometer measures the sagittal radius of curvature $r_{\mathrm{s} 1}$ for the defined point.

To find $h_{1}$ for radius $r_{\mathrm{s} 1}$

$i=r_{\mathrm{s} 1} / 2 \times \sin 18^{\circ}$

$\operatorname{sag}=r_{\mathrm{s} 1} / 2-\sqrt{\left(\left(r_{\mathrm{s} 1} / 2\right)^{2}-i^{2}\right)}$

$i / h_{1}=100 / 100-\left(r_{\mathrm{s} 1} / 2+\mathrm{sag}\right)$

$h_{1}=i\left(100-\left(r_{\mathrm{s} 1} / 2+\mathrm{sag}\right)\right) / 100$

The perpendicular distance from the optical axis of the keratometer $h_{1}$ defines the point being measured.

When the surface is tilted 18 degrees, for example, the situation is as described in Figure 2.

It is initially assumed that the point on the surface that is being measured coincides with the point where the keratometer axis intersects the surface. This is an oversimplification and represents a starting point for the method. The two mire extremities will be more peripheral and more central in relation to the intersection point. So one mire extremity image will be formed by a flatter and one formed by a steeper part of the surface. The average of these two should produce a radius measurement close to the intersection radius $r_{\mathrm{s} 2}$ at $h_{2}$.

$h_{2}=r_{\mathrm{s} 2} \cdot \sin 18^{\circ}$

Now the problem with this result is that the values derived for $h_{1}$ and $h_{2}$ are approximations 


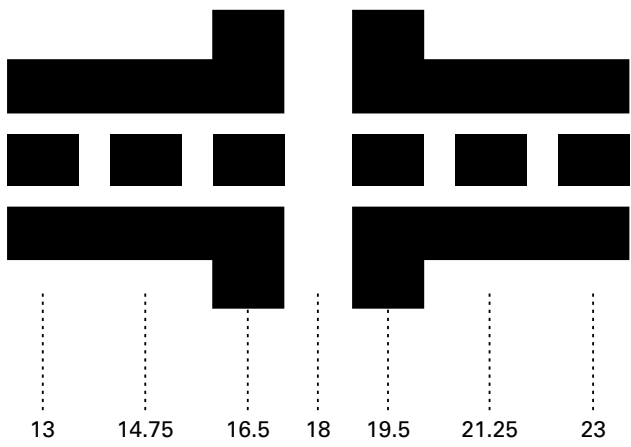

Figure 3 Diagram showing the hollow cross mire target of the Zeiss telecentric keratometer. The numbers refer to the angular subtense of the centre of each block from the instrument optical axis.

owing to assumptions made in deriving equations (1) and (2).

However, Sheridan and Douthwaite ${ }^{11}$ used the equation:

$r_{\mathrm{s}}^{2}=r_{\mathrm{o}}^{2}+(1-p) h^{2}$

which can be rewritten as

$\left.h=\sqrt{(}\left(r_{\mathrm{s}}^{2}-r_{\mathrm{o}}^{2}\right) /(1-p)\right)$

So if we take the apical radius and the $\mathrm{p}$ value of the surface according to the Talysurf analysis and then substitute the measured radius from the keratometer, we can calculate the actual value of $h$.

Previous investigations into corneal topography have suggested that corneal sections approximate to conic sections. It is extremely unlikely that the corneal section will be an exact conic section in an individual cornea and so a technique that relies on only two radius measurements for a given meridian is unlikely to allow the derivation of a representative corneal apical radius and $\mathrm{p}$ value. It was therefore decided to develop a technique for deriving these two parameters from keratometric measurements taken at a number of different points on the aspherical surfaces employed in this investigation.

The Zeiss telecentric keratometer possesses a mire with a solid and hollow cross (see Figure 3).

The hollow cross contains six small squares and there is also the centre of the cross itself. These reference points can be used as seven fixation targets producing seven different angles of tilt for the human cornea giving eight radius measurements. The angles produced by these fixation targets were extracted from the instrument manufacturer's literature kindly provided by Zeiss.

A holder was manufactured to hold the test buttons perpendicular to the instrument axis and to allow various angles of horizontal tilt. These tilt angles were 13, 14.75, 16.5, 18, $19.5,21.25$, and 23 degrees, which equate to the angles subtended by the fixation points along the hollow cross mire. As each surface was mounted in the holder the clockwise and anticlockwise values for $18^{\circ}$ of tilt were checked to ensure that similar radii were recorded. A difference less than $0.05 \mathrm{~mm}$ in the keratometric radius between the $18^{\circ}$ clockwise and anticlockwise results was accepted as an indicator that the surface tilt was zero for conventional keratometry.

Four conventional keratometry measurements were performed on the engraved horizontal meridian of each aspherical surface. The average radius was recorded. Measurements were then made in each of the tilted positions, keratometry being performed alternately using clockwise and anticlockwise rotations with two measurements recorded in each direction. Any remaining inaccuracy due to any slight error in surface placement was minimised by recording the radius for any tilt angle as the average of the four values. Equations (1) and (2) were used to calculate initial values for $h$ and equation (3) was used to calculate the actual values for $h$ for each tilt angle. A scatter plot of actual values for $h$ versus the $h$ values derived by keratometric measurement was plotted, with a separate scatter plot being drawn for each tilt angle. The function was found to be different for each tilt angle but was linear in every case. The regression equations were used to provide a correction factor to change the approximate value of $h$ to a corrected value for each angle of tilt. For each surface point we have a sagittal radius measurement $\left(r_{\mathrm{s}}\right)$ centred on that point which is defined by its corrected perpendicular distance from the instrument optical axis $(h)$.

Equation (4) was the equation used by Douthwaite $^{12}$ to assess the accuracy of the EyeSys VK.

$r_{\mathrm{s}}^{2}=r_{\mathrm{o}}^{2}+(1-p) h^{2}$

The apical radius $r_{\mathrm{o}}$ and the $\mathrm{p}$ value $p$ are constants of the surface. Equation (4) is therefore the equation for a straight line graph.

Plotting a graph of perpendicular distance squared ( $h^{2}$ on the abscissa) versus radius squared $\left(r_{\mathrm{s}}^{2}\right.$ on the ordinate) will allow the calculation of apical radius (the square root of the intercept of the regression line on the radius squared axis) and the $p$ value (1- slope of the regression line).

Graphs were plotted for each of the 22 buttons and the results were compared with the apical radius and $\mathrm{p}$ value derived by Talysurf analysis.

It must be noted that the keratometric images of the two mire extremities were not simultaneously in focus at the greater tilt angles in the more aspheric surfaces. In these conditions, the keratometer focus was adjusted for the best overall focus before alignment was attempted. Also subsequent analysis revealed that the more peripheral measurements did not contribute to the accuracy of the final result for the aspheric buttons. It was, therefore, decided to make the 18 degree tilt the maximum angle for recording radii on human corneas. This has the added advantage that the topometric measurements will cover approximately the same corneal area as those of the EyeSys VK. 

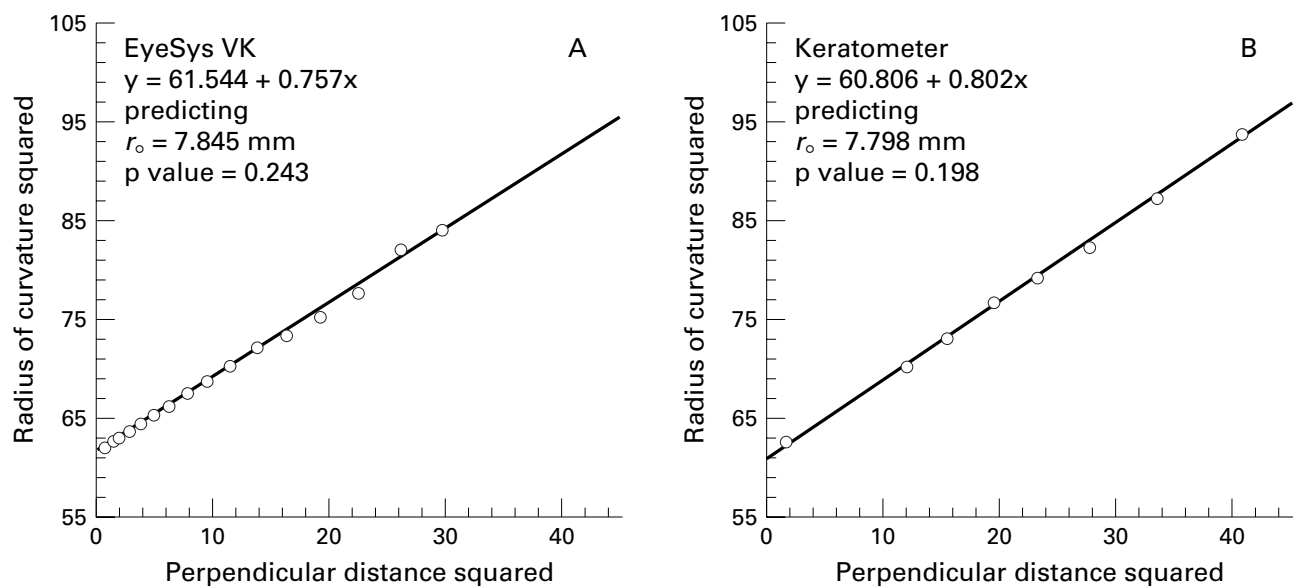

Figure 4 Typical radius squared versus distance squared graphs for one of the 22 aspherical surfaces. (A) The scatter plot derived from the EyeSys VK measurements. (B) The scatter plot derived from the Zeiss keratometric measurements. The Talysurf analysis for this surface gave an apical radius of $7.800 \mathrm{~mm}$ and a $p$ value of 0.212 .

The same 22 surfaces were examined by the EyeSys VK (software version 3.2), following the manufacturer's recommendations. The VK tabular data display was used to draw a scatter plot of perpendicular distance squared versus radius squared as above averaging the paired values from the two semimeridians for both the radii and the perpendicular distances. The apical radius and the $\mathrm{p}$ value were derived from this scatter plot. ${ }^{12}$
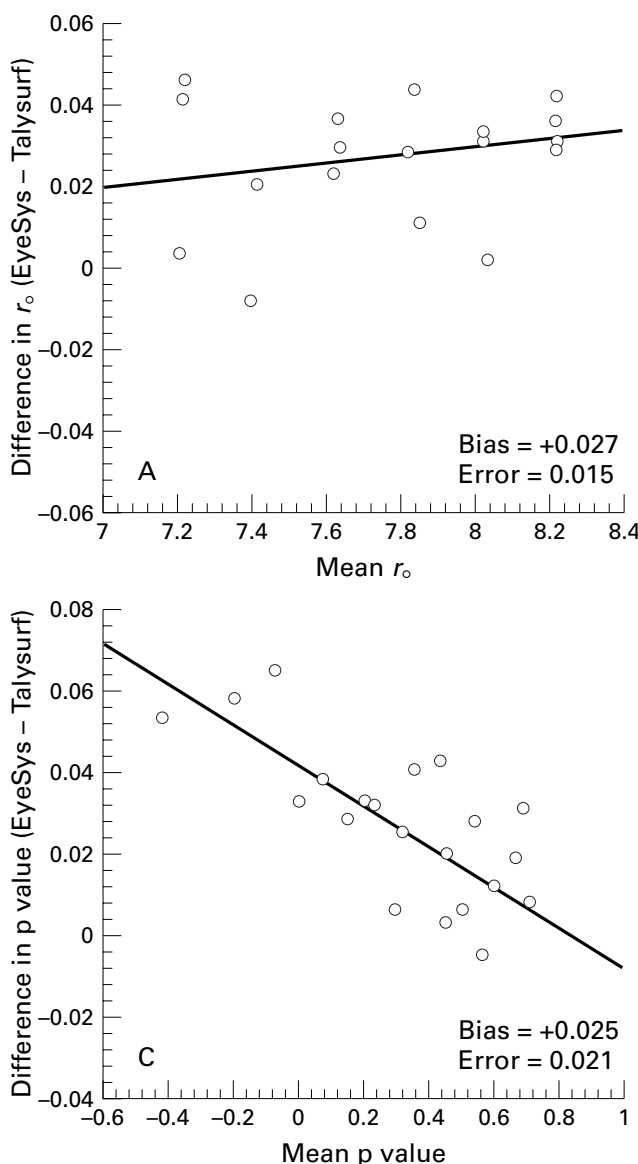

The topometry technique and the EyeSys VK were finally used on the right eyes of 30 adult human subjects. There were 15 males and 15 females. Every subject gave informed consent. All subjects were free of ocular pathology and had refractive errors within 6.00 $\mathrm{D}$ of spherical and $2.00 \mathrm{D}$ of astigmatic error. Once again, apical radii and $\mathrm{p}$ values were calculated from the measurements for the near horizontal principal meridian. The examina-
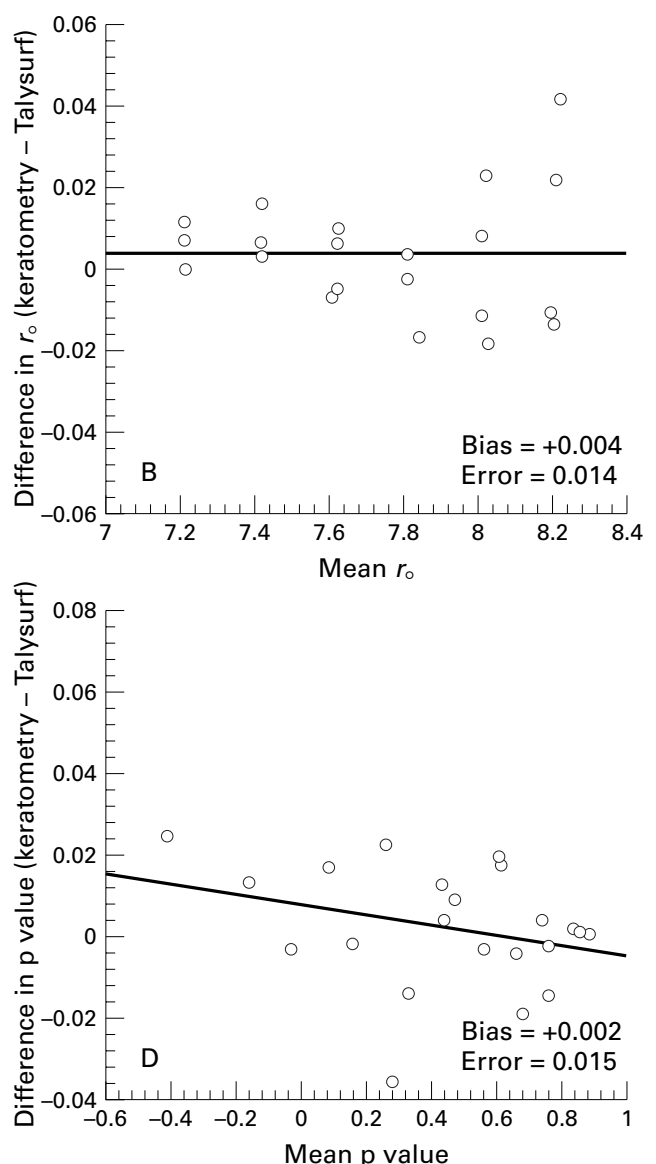

Figure 5 (A) A graph of the mean apical radius ((Talysurf + EyeSys)/2) versus the apical radius difference. (B) $A$ graph of the mean apical radius ((Talysurf + keratometer)/2) versus the apical radius difference. (C) A graph of the mean $p$ value ((Talysurf + EyeSys)/2) versus the $p$ value difference. (D) A graph of the mean $p$ value ((Talysurf + keratometer $) / 2)$ versus the $p$ value difference. The bias is the mean difference and the error is the standard deviation of the difference. 

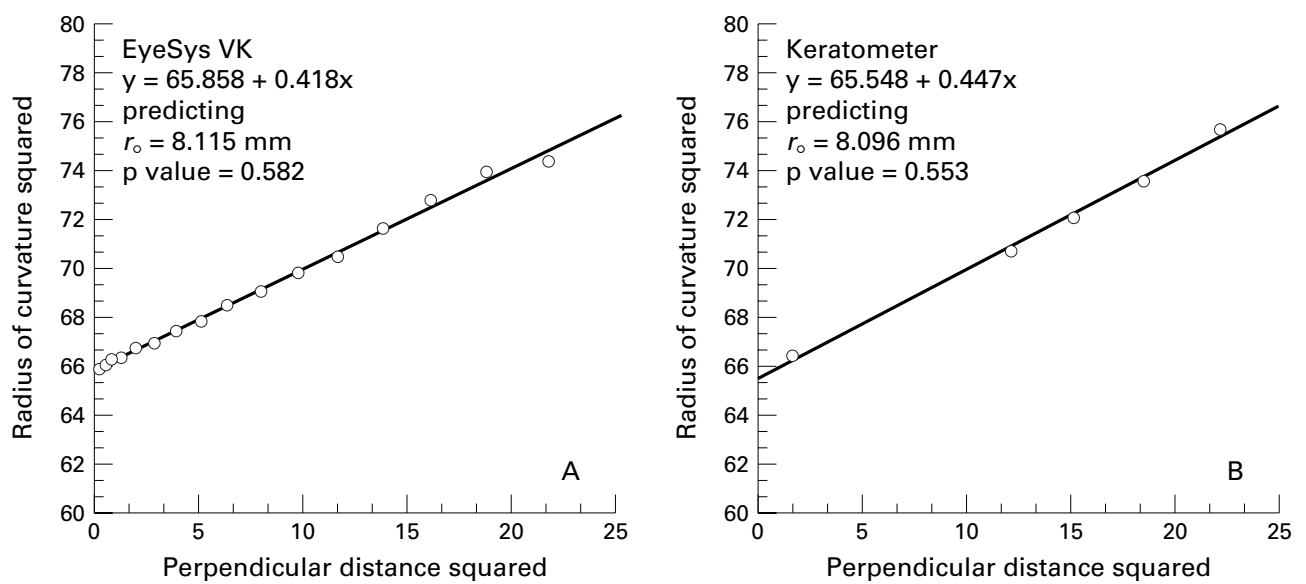

Figure 6 (A) Typical radius squared versus distance squared graphs for one of the human corneas. The scatter plot derived from the EyeSys VK measurements. (B) The scatter plot derived from the Zeiss keratometric measurements.

tion of the human corneas allowed a comparison of the topometry method results with those of the EyeSys VK.

\section{Results}

The Kolmogorov-Smirnov d test indicated that parametric statistics could be used for the data analysis.

Figure 4 illustrates the scatterplots acquired for one of the aspherical surfaces. The graphs plot the perpendicular distance squared versus sagittal radius squared for the EyeSys VK and for the keratometer.

The theoretical result for the graphs, assuming that an elliptical section has been measured, is one where all data points lie on a straight line. The square root of the intercept on the ordinate gives the apical radius. The $\mathrm{p}$ value is acquired by subtracting the slope of the regression line from unity. Both instruments have produced data points that lie very close to the regression line. However, there is a disagreement for both the apical radius and the $\mathrm{p}$ value. The Talysurf measurements for the surface in Figure 4 produced an apical radius result of $7.80 \mathrm{~mm}$ and 0.212 for the $\mathrm{p}$ value.

The results for all 22 buttons were compared using the EyeSys and the keratometric results. A final comparison was made with the Talysurf analysis. Altman and Bland ${ }^{13}$ recommend plotting a graph of the mean $((\mathrm{A}+\mathrm{B}) / 2)$ against the difference $(A-B)$ in order to compare two methods of measurement. Figure 5 illustrates the differences in the apical radii and $p$ values derived by the three methods of measurement.

The graphs show that both instruments are deriving apical radii and $p$ values close to those of the Talysurf analysis with the regression lines suggesting a better agreement between the Talysurf and the keratometer, than the Talysurf and EyeSys results. Altman and Bland $^{13}$ state that the mean of the differences will give the relative bias and the standard deviation of the differences is the estimate of error.

The keratometer results produce values for both surface parameters that are very slightly greater than those of the Talysurf. Greater differences are found for the values derived from the VK. A repeated measures ANOVA for both apical radius $(F ; 2,42=42.421, \mathrm{p}=0.000)$ and $\mathrm{p}$ value $(F ; 2,42=19.201, \mathrm{p}=0.000)$ and post hoc Scheffe tests revealed significant differences between results for the EyeSys compared with both Talysurf and the keratometer ( $p=0.000$ in all cases). The keratometer, however, did not produce significantly different parameters from those predicted by Talysurf analysis in either case (apical radius $\mathrm{p}$ $=0.518, \mathrm{p}$ value $\mathrm{p}=0.869$ ).

When we go on to consider the results of the human corneas, we do not have the advantage of a Talysurf result. The only comparison that can be made is a comparison of the $\mathrm{VK}$ and keratometric results. Figure 6 illustrates the scatterplots produced by the two methods of measurement on subject number 30 .

The group mean values for apical radius and $\mathrm{p}$ value derived by the two instruments are 7.832 (SD 0.251) $\mathrm{mm}$ for the keratometer and $7.859(0.732) \mathrm{mm}$ for the VK. The respective $\mathrm{p}$ values are $0.251(0.075)$ and $0.255(0.106)$.

A related $t$ test on the human corneas indicates a significant difference between the $\mathrm{VK}$ and the keratometer $(t=4.861, \mathrm{p}=0.000)$ for apical radius and no significant difference $(t=0.722, \mathrm{p}=0.476)$ for the $\mathrm{p}$ value.

\section{Discussion}

The aspheric button scatter plots of distance squared versus radius squared, illustrated in Figure 4, indicate that both the EyeSys VK and the keratometer produced measurements which were very close to the Talysurf result and that the data points were all very close to the regression line. There is no significant difference between the keratometric and the Talysurf results. The VK, however, estimated slightly longer apical radii (bias $=0.027 \mathrm{~mm}$ ) and slightly less asphericity (bias $=0.025$ ) which were statistically significantly different from the other two methods of measurement.

When we examine human corneas that are known to approximate to the conic sections, ${ }^{1415}$ we unfortunately do not have the benefit of known surface parameters with which to compare our results. Bland ${ }^{16}$ suggests the use of the one sample $t$ method as a means of determining how much higher or lower one piece of equipment may read in comparison 
with another. On the basis of this analysis with a probability of $95 \%$, the keratometer underreads from 0.015 to $0.037 \mathrm{~mm}$ for apical radius and for $\mathrm{p}$ value the keratometer may underread by 0.031 or overread by 0.015 when compared with the VK.

Comparing the Talysurf analysis with the VK result for the buttons shows that the Talysurf will underread from 0.021 to $0.034 \mathrm{~mm}$ for apical radius and will underread from 0.015 to 0.034 for the $p$ value. The above suggests that the keratometric result may be more accurate than that achieved by the VK.

The corneal topography could be described by establishing an apical radius and $\mathrm{p}$ value for each of the two principal meridians. All that is required is a series of fixation targets incorporated into the mire. The advantage of incorporating the fixation targets into the mire is that the corneal tilt will always be along the principal meridians no matter what their orientation.

The basic program in the appendix is the one that was used to determine the apical radius and $\mathrm{p}$ value with the keratometer. The technique holds promise as a keratometric method for measuring human corneal asphericity as well as curvature.

\section{Appendix}

The basic program used to calculate the apical radius and the $p$ value from the topometric measurements

10 REM determination of $p$ and ro from the zeiss

11 REM eccentric $\mathrm{k}=$-readings on human corneas using topometry throughout

15 CLEAR : CLS

20 REM telecentric keratometer

30 LET $1=100$ : REM 1 is object distance

40 LET a $=.3142:$ REM angle $(\mathrm{rad})$ of half the mire

50 PRINT : PRINT "CALCULATION OF RO AND P"

60 PRINT : PRINT "ENTER THE CONVEN-

TIONAL K-READING": INPUT rs1

70 LET $\mathrm{rs} 1=\mathrm{rs} 1 / 2$

80 LET i $=$ rs 1 * $\operatorname{SIN}(.3142)$

90 LET sag $1=$ rs1 $-\mathrm{SQR}\left(\mathrm{rs} 1^{\wedge} 2-\mathrm{i}^{\wedge} 2\right)$

$100 \operatorname{LET~h} 1=((100-(\mathrm{rs} 1+\operatorname{sag} 1)) / 100){ }^{*} \mathrm{i}$

105 REM correction

106 LET $\mathrm{h} 1=\mathrm{h} 1$ * $.68717+.48583$

110 PRINT : PRINT "VERTICAL CORNEAL

DISTANCE h1 IS "; h1; " mm"

115 LET rs $1=\mathrm{rs} 1$ * 2

120 REM radius measurements when the cornea is tilted

$125 \mathrm{FOR} \mathrm{i}=1 \mathrm{TO} 5$

$130 \mathrm{IF} \mathrm{i}=1$ THEN LET rka $=\mathrm{rs} 1:$ LET dista $=\mathrm{h} 1:$ GOTO 630

280 REM angle of fixation a2

300 IF $\mathrm{i}=2$ THEN PRINT : PRINT "ENTER THE RADIUS FOR A 13 DEGREE FIXATION":

INPUT rs2

$310 \mathrm{IF} \mathrm{i}=2$ THEN LET a $2=.2269:$ LET $\mathrm{h} 2=\mathrm{rs} 2$ $\star \operatorname{SIN}(\mathrm{a} 2)$

320 IF $\mathrm{i}=2$ THEN LET rp2 = rs2: LET hp2 = h2:

LET hp $2=\mathrm{h} 2 \star 1.878-.0799$

330 IF $\mathrm{i}=2$ THEN LET rka $=$ rp $2:$ LET dista $=\mathrm{hp} 2$

335 IF $\mathrm{i}=3$ THEN PRINT : PRINT "ENTER

THE RADIUS FOR A 14.75 DEGREE FIXATION":

INPUT rs2

$340 \mathrm{IF} \mathrm{i}=3$ THEN LET a $2=.2574:$ LET h2 $=$ rs 2

* $\operatorname{SIN}(\mathrm{a} 2)$

350 IF $\mathrm{i}=3$ THEN LET rp $3=$ rs2: LET hp3 = h2:

LET hp $3=\mathrm{h} 2$ * $1.659+.29589$

360 IF $\mathrm{i}=3$ THEN LET rka $=$ rp $3:$ LET dista $=\mathrm{hp} 3$

$365 \mathrm{IF} \mathrm{i}=4$ THEN PRINT : PRINT "ENTER

THE RADIUS FOR A 16.5 DEGREE FIXATION":

INPUT rs2
$370 \mathrm{IF} \mathrm{i}=4$ THEN LET $\mathrm{a} 2=288:$ LET $\mathrm{h} 2=\mathrm{rs} 2$ * $\operatorname{SIN}(\mathrm{a} 2)$

$380 \mathrm{IF} \mathrm{i}=4$ THEN LET $\mathrm{rp} 4=\mathrm{rs} 2:$ LET hp $4=\mathrm{h} 2:$ LET hp $4=\mathrm{h} 2 \star 1.6212+.35028$

390 IF $\mathrm{i}=4$ THEN LET rka $=$ rp $4:$ LET dista $=$ hp 4 $395 \mathrm{IF} \mathrm{i}=5$ THEN PRINT : PRINT "ENTER THE RADIUS FOR A 18 DEGREE FIXATION": INPUT rs2

$400 \mathrm{IF} \mathrm{i}=5$ THEN LET a $2=3142: \mathrm{LET} \mathrm{h} 2=\mathrm{rs} 2$ $\star \operatorname{SIN}(\mathrm{a} 2)$

$410 \mathrm{IF} \mathrm{i}=5$ THEN LET rp $5=$ rs $2:$ LET hp $5=\mathrm{h} 2$ :

LET hp $5=\mathrm{h} 2 \star 1.4729+.74177$

$420 \mathrm{IF}$ i $=5$ THEN LET rka $=$ rp5: LET dista $=$ hp 5

630 REM calculating the sigma values for regression line

640 LET dista $=$ dista ^2: LET ax $=a x+$ dista

650 LET $\mathrm{sx}=\mathrm{ax}$

$660 \mathrm{LET}$ rka $=\mathrm{rka}^{\wedge} 2: \mathrm{LET}$ ar $=\mathrm{ar}+\mathrm{rka}$

670 LET sy $=$ ar

680 LET $x y=($ dista $*$ rka $):$ LET $s x y=s x y+x y$

$690 \operatorname{LET~xt}=\operatorname{dista}^{\wedge} 2:$ LET sxt $=\mathrm{sxt}+\mathrm{xt}$

700 LET $\mathrm{n}=\mathrm{i}$

710 NEXT i

850 PRINT : PRINT "RADIUS PERP DISTANCE”

860 PRINT : PRINT rs 1, h1

870 PRINT rp2, hp2: PRINT rp3, hp3: PRINT rp4, hp4: PRINT rp5, hp5

880 PRINT rp6, hp6: PRINT rp7, hp7: PRINT rp8, hp 8

920 REM sub routine for calculating the regression line

930 LET $\mathrm{b}=(\mathrm{n}$ * $\mathrm{sxy}-\mathrm{sx}$ * $\mathrm{sy}) /(\mathrm{n}$ * $\mathrm{sxt}-\mathrm{sx}$ * $\mathrm{sx})$

940 LET a $=(\mathrm{sy}-\mathrm{b} \star \mathrm{sx}) / \mathrm{n}$

$945 \mathrm{LET}$ a $=$ SQR $(\mathrm{a})$

950 PRINT " THE VERTEX RADIUS IS ";

INT $(1000$ * $\mathrm{a}+.5) / 1000 ; " \mathrm{~mm}$ "

955 LET $\mathrm{b}=1-\mathrm{b}$ :

960 PRINT ” THE P-VALUE IS "; $\operatorname{INT}(1000$ * $b+$ .5)/1000

970 PRINT : PRINT “ANOTHER CORNEA ? $(\mathrm{Y} / \mathrm{N}) "$ : INPUT q $\$$

980 IF q\$ = "Y" OR q = “y” THEN GOTO 10

990 STOP

1 Antalis JJ, Lembach RG, Carney LG. A comparison of the TMS-1 and the corneal analysis system for the analysis of abnormal cornea. CLAO F 1993;19:58-63.

2 Maeda N, Klyce SD, Smolek MK. Comparison of methods for detecting keratoconus using videokeratography. Arch for detecting keratoconus usin

3 Douthwaite WA, Pardhan S. Comparison of a videokeratoscope and an autokeratometer as predictors of the scope and an autokeratometer as predictors of the lenses. Ophthalmic Physiol Opt 1997;17:409-13.

4 Lam AKC, Douthwaite WA. Three month study of changes in the cornea after computer-determined and conventionally determined contact lens fitting. Ophthalmic Physiol Opt 1994;14:59-64.

5 Bonnet R. Stereophotogrammetric de la cornee humaine. Rev Optique 1959;38:447-60.

6 Arffa RC, Warnicki JW, Rehkopf PG. Corneal topography using rasterstereography. Refract Corneal Surg 1989;5:41417.

7 Belin MW, Litoff D, Strods SJ, et al. The PAR technology corneal topography system. Refract Corneal Surg 1992;8: 88-96.

8 Belin MW, Zloty P. Accuracy of the PAR corneal topography system with spatial alignment. CLAO $f$ 1993;19:64-8.

9 Bonnet R, Cochet D. New method of topographical ophthalmometry -its theoretical and clinical applications. ophthalmometry-its theoretical and clin

10 Wilms KH, Rabbetts RB. Practical concepts of corneal topometry. Optician 1977; Sept 16:7-13.

11 Sheridan M, Douthwaite WA. Corneal asphericity and refractive error. Ophthal Physiol Opt 1989;9:235-7.

2 Douthwaite WA. EyeSys corneal topography measurement applied to calibrated ellipsoidal convex surfaces. $\mathrm{Br} F \mathrm{Oph}$ thalmol 1995;79:797-801

13 Altman DG, Bland JM. Measurement in medicine: the analysis of method comparison studies. Statistician 1983; 32:307-17.

14 Mandell RB, StHelen R. Mathematical models of the general corneal contour. Br F Physiol Opt 1971;26:183-97.

15 Burek $\mathrm{H}$, Douthwaite WA. Mathema (he general corneal surface. Ophthalmic Physiol Opt 1993;13:

16 Bland M. An introduction to medical statistics. Oxford: Oxford Medical Publications, 1989:280-2. 